\title{
Stability of water-in-oil emulsion in liquid membrane prospect
}

\author{
Norela Jusoh ${ }^{a}$, Norasikin Othman ${ }^{a, b}$, * \\ a Department of Chemical Engineering, Faculty of Chemical and Energy Engineering, Universiti Teknologi Malaysia, 81310 UTM Johor Bahru, \\ Johor, Malaysia \\ b Centre of Lipids Engineering and Applied Research (CLEAR), Ibnu Sina Institute of Scientific and Industrial Reasearch, Universiti Teknologi \\ Malaysia, 81310 UTM Johor Bahru, Johor, Malaysia \\ * Corresponding author: norasikin@cheme.utm.my
}

\section{Article history}

Received 25 May 2016

Accepted 16 October 2016

\begin{abstract}
Emulsion liquid membrane (ELM) process have shown a great potential in wide application of industrial separations such as in removal of many chemicals, organic compounds, metal ions, pollutants and biomolecules. ELM promotes many advantages including simple operation, nonequilibrium mass transfer, high selectivity, low energy requirements, and simultaneous extraction and stripping process in a single step process. New development in ELM system incorporated with a green solvent (palm oil) was attempted instead of using commercial organic solvent. The important aspects must be considered for a successful ELM process is the stability of the liquid membrane. In the current work the effect of various parameter which are organic to internal ratio, emulsifying speed, surfactant concentration, and emulsifying time on green ELM stability were investigated. The results show that the most stable emulsion was observed at 3:1 organic to internal ratio, $7000 \mathrm{rpm}$ speed, $3 \%(\mathrm{w} / \mathrm{v})$ surfactant concentration, and 5 minute emulsifying time. The emulsion obtained was stable up to $1 / 2$ hour and sufficient and sufficient for extraction process. Therefore, green solvent has high potential to be applied in emulsion liquid membrane process.
\end{abstract}

Keywords: emulsion liquid membrane, green, stability, palm oil

\section{INTRODUCTION}

Liquid membrane is a system in which the organic liquid divides the aqueous feed and the product phases. It consists of a thin film of organic reagent. One of the liquid membrane types is emulsion liquid membrane (ELM), which is introduced as alternative technique to the separation process.

Emulsion liquid membrane process consist four main stages: 1) emulsification, 2) dispersion and extraction, 3) settling, and 4) demulsification. Firstly, emulsion formed from droplet of strip solution dispersed in liquid membrane in the presence of surfactant After that, the emulsion is dispersed into external feed phase containing solute of interest. When the extraction is complete, the emulsion phase is separated from the external phase, and broken to recover the membrane phase.

ELM fulfills the promise of providing high transport efficiencies due to high interfacial area for mass transfer, economical, low energy consumption, combination of extraction and stripping process, efficient for low solute concentration, and requirement small quantity of solvent [1-3]. With these advantages the ELM process becomes an attractive alternative and a promising technology to be applied in research and industry.

Emulsion liquid membrane process was widely studied for industrial separations such as in removal of phenol from wastewater $[4,5]$, recovery of palladium from electroplating wastewater $[6,7]$, extraction of silver from wastewater [8-10], lignin recovery from pulping wastewater [11], and removal of cadmium [12]. Recent development in emulsion liquid membrane process is in downstream processing of bio-succinic acid due to increasing demand of succinic acid as building block chemical [13,14].

However, the main drawback related to ELM is the emulsion instability, which is governed by emulsion breakage. Membrane breakage in ELM systems includes the rupture of emulsion and leakage of internal stripping solution and extracted solute to the external phase. These cause the decrease in the volume of the stripping phase [15], resulting in the membrane breakage. As a result, concentration gradient, which is the driving force for mass transfer reduces and increase the feed concentration, thereby lowering the extraction efficiency. The instability can be attributed to the emulsion formulation in terms of the choice of carrier, diluent, surfactant, stripping agent, and emulsification procedure. The carrier should be selective to the target succinic acid solute while the stripping agent and the type of surfactant must be properly chosen to minimize the cotransport of water during extraction process.

In ELM process, the diluent is very important, since it is the major component of the membrane phase and is crucial for emulsion stability. Diluent should have a low solubility in water in order to create the membrane phase, it should also provide high carrier solubility, high-boiling point, non-toxic and relatively cheap [16, 17]. Most studies have commonly used kerosene as organic diluent due to its viscosity, readily availability and non-polar character. However, kerosene is not considered environmentally friendly and harmful to human. Alternatively, palm oil can be chosen as renewable organic diluent, as it is readily available and may contain natural surfaceactive agents, which improve the stability of an emulsion [18]. In 
addition, palm oil was proven to work well in extraction of heavy metal and phenol $[19,20]$.

In this study, the main focus was to investigate primary water-inoil emulsion stability using Amberlite LA-2 as carrier, palm oil as diluent, sodium carbonate as aqueous stripping agent, and Span 80 as surfactant. Several operating parameters were investigated including organic to internal ratio, emulsifier speed, emulsifying time, surfactant type, and surfactant concentration.

\section{EXPERIMENTAL}

\section{Materials}

Palm oil as diluent is ordinary cooking oil (BURUH) from Lam Soon Edible Oils. Amberlite LA-2 as carrier was a mixture of straight chain secondary amine (M=374 $\mathrm{g} / \mathrm{mol})$ and purchased from MERCK. Solid sodium carbonate $\left(\mathrm{Na}_{2} \mathrm{CO}_{3}\right)(99 \%$ assay) used as internal stripping solution was obtained from Merck. Sorbitan monooleate (Span 80) (with more than 60\% oleic acid) is used as surfactant and purchased from Sigma Aldrich. All these reagents and solutions were used directly as received without further purification.

\section{Methods}

Organic liquid membrane solution containing Amberlite LA-2 and Span 80 (1 to $7 \%$ ) in palm oil and aqueous $\mathrm{Na}_{2} \mathrm{CO}_{3}$ stripping solution (1 to 3 organic to internal ratio) was emulsified at different speeds (5000 rpm to $12000 \mathrm{rpm}$ ) for different times ( 3 to 20 minutes) using motor driven homogenizer (Heidolph Silent Crusher M) to obtain primary emulsion. Immediately after emulsification, the emulsion was poured into a $10 \mathrm{~mL}$ measuring cylinder. The stability of the emulsion was determined by recording the volume of aqueous phase separated as a function of time. More aqueous phase separated indicate the emulsion is unable to remain dispersed and less stable. The aggregation of the emulsion droplets was directly observed under a polarized microscope (Olympus CX31). Meanwhile, number of droplets formed was counted within circle of $30 \mu \mathrm{m}$ radius and size of droplet was determined by taking average size of 30 droplets using VImage 2014 software. The size of the droplet was represented as Sauter mean diameter $\left(d_{32}\right)$, defined in Equation 1:

$$
d_{32}=\frac{\sum\left(n_{i} \cdot d_{i}^{3}\right)}{\sum\left(n_{i} \cdot d_{i}^{2}\right)}
$$

in which $n$ is the number of $d$ diameter droplets.

\section{RESULTS AND DISCUSSION}

\section{Effect of emulsion composition}

Effect of liquid membrane composition on emulsion stability was investigated by varying the ratio of organic phase to internal phase ratio and was summarized in Table 1. 1:1 ratio shows the most unstable emulsion, where $26 \%$ of emulsion was separated in 10 minutes, indicating the internal phase did not remained dispersed in the emulsion. This is due to the inherent nature of oil and the accompanying stearin as natural surfactant in the oil which alter the oil/water interface, hence reduce the stability [18]. Data from Table 1 shows lowest droplet counted for 1:1 ratio which is 50 droplets, showing the low droplet formation. According to Table 1, stability of water-in-oil improved when increasing the ratio to $2: 1$ and 3:1. This can be attributed to the increasing membrane layer around the droplets. The results is in line with Jilska and Geoff [21], who reported that stability of emulsion increase when reducing the volume fraction of internal phase. Besides, increasing the organic fraction also increase the Span 80 content in the emulsion, reducing more interfacial tension, resulting in more droplets formed. This can be seen in Table 1, where droplet counts are 100 and 130 for 2:1 and 3:1 O:I ratio respectively. In addition, mechanical resistance of the membrane also increased at higher organic fraction, thus prevent coalescent of the dispersed droplet. Meanwhile, 2.5 to $4.0 \mu \mathrm{m}$ of droplet size was recorded, indicating the size is within the range of standard droplet size [22]. In general, larger droplet increase the emulsion instability because the droplet easy to coalesce. However, in this study largest droplet was observed at the most stable 3:1 O/I ratio, indicating the stability not necessarily depend on the droplet size. Hence, $3 / 1$ of $\mathrm{O} / \mathrm{I}$ ratio is highly preferable to produce a stable emulsion.

Table 1 Effect of emulsion composition on emulsion stability.

\begin{tabular}{|c|c|c|c|c|c|}
\hline \multirow{2}{*}{ O/I } & \multicolumn{2}{|c|}{ Aqueous phase separated (\%) } & \multirow{2}{*}{$\begin{array}{c}\text { Droplet } \\
\text { count }\end{array}$} & $\begin{array}{c}\text { Droplet } \\
\text { size }(\mu \mathrm{m})\end{array}$ \\
\cline { 2 - 4 } & $10 \mathrm{~min}$ & $30 \mathrm{~min}$ & $60 \mathrm{~min}$ & & 5.5 \\
\hline $1: 1$ & 26 & 36 & 40 & 50 & 2.5 \\
\hline $2: 1$ & 10 & 16 & 30 & 100 & 3.9 \\
\hline $3: 1$ & 0 & 1 & 5 & 130 & 4.0 \\
\hline
\end{tabular}

Diluent: palm oil; [Amberlite LA2]: $0.05 \mathrm{M}$; $\left[\mathrm{Na}_{2} \mathrm{CO}_{3}\right.$ ]: $0.5 \mathrm{M}$; speed: 5000 rpm; emulsifying time: 5min; Span 80: $3 \% \mathrm{w} / \mathrm{v}$; HLB: 4.3

\section{Effect of homogenizer speed}

Table 2 presented the effect of homogenizer speed on the performance of water-in-oil stability. Increasing homogenizer speed from 5000 to $7000 \mathrm{rpm}$ increase the emulsion stability. This is because increasing the homogenizer speed provides better mixing and reduce more interfacial tension between the aqueous and organic phase which generates more droplet produced, hence stabilize the emulsion. Data in Table 2 proved that more droplets was formed with $7000 \mathrm{rpm}$ which is 150 droplets compared to 130 droplets with 5000 rpm. A study by Sulaiman et al. [8] also found that higher homogenizer speed increase emulsion stability. Further increase the speed to $9000 \mathrm{rpm}$ increase the droplet formation to 180 droplets. However, the phase separation data shows that the stability is lower than that of $7000 \mathrm{rpm}$. Rapid mixing causes the droplets tend to coalesce among each other, thus enlarging their size as shown in Table 2 where the size maximum size was recorded at $5.8 \mu \mathrm{m}$ which leading to the breakage of the droplet. At $12000 \mathrm{rpm}$ homogenizer speed, a highly viscous, "mayonnaise-like" emulsion was formed. This is due to foaming mechanism, where air-bubbles are incorporated into the emulsion phase, and lead to a more rigid system. This result is in line with a study reported by Bjorkegren and Karimi [19], who also found that higher speed resulted in highly viscous emulsion. Therefore, homogenizer speed at $7000 \mathrm{rpm}$ is preferable in this study in producing stable emulsion.

Table 2 Effect of homogenizer speed on emulsion stability.

\begin{tabular}{|c|c|c|c|c|c|}
\hline \multirow{2}{*}{$\begin{array}{c}\text { Speed } \\
(\mathbf{r p m})\end{array}$} & \multicolumn{2}{|c|}{ Aqueous phase separated (\%) } & \multirow{2}{*}{$\begin{array}{c}\text { Droplet } \\
\text { count }\end{array}$} & $\begin{array}{c}\text { Droplet } \\
\text { size }(\boldsymbol{\mu m})\end{array}$ \\
\cline { 2 - 4 } & $\mathbf{1 0} \mathbf{~ m i n}$ & $\mathbf{3 0} \mathbf{~ m i n}$ & $\mathbf{6 0 m i n}$ & & 4.0 \\
\hline 5000 & 0 & 1 & 5 & 130 & 4.9 \\
\hline 7000 & 0 & 1 & 3 & 150 & 5.8 \\
\hline 9000 & 0 & 1 & 6 & 180 & 5.2 \\
\hline 12000 & 0 & 1 & 1 & 110 & \\
\hline
\end{tabular}

Diluent: palm oil; [Amberlite LA2]: 0.05M; $\left[\mathrm{Na}_{2} \mathrm{CO}_{3}\right.$ ]: $0.5 \mathrm{M} ; \mathrm{O} / \mathrm{I}: 3 / 1$; emulsifying time: $5 \mathrm{~min}$; Span 80: $3 \%$ w/v; HLB: 4.3

\section{Effect of emulsifying time}

The effect of emulsification time on water-in-oil emulsion stability is shown in Table 3. The result shows that at 3 minutes emulsification time, about $2 \%$ of aqueous phase was separated from the emulsion in 10 minutes. This indicates that short emulsifying time produce unstable emulsion because of the mixture of organic membrane and aqueous internal solution was not well homogenized. As a result, the configuration of surfactant at the interfacial area is unorganized, less interfacial tension is reduced, and thus larger droplets $(11.2 \mu \mathrm{m})$ were formed and make it easy to break. Additions of emulsification time to 5 minutes produced more stable emulsion, where it starts to break after 30 minutes. This is due to smaller internal droplets formed at $4.9 \mu \mathrm{m}$ thus leading to stable emulsion. However, further increase the emulsification time up to 10 minutes and above, it 
will decrease the water-in-oil emulsion stability because the emulsion was exposed to the high shear longer and causing emulsion breakage. The breakage phenomena associated with prolonged emulsification time also observed by Othman et al. [23]. On the other hand, 100 and 80 droplets was counted for 10 and 15 minutes emulsifying time respectively, compared to 150 droplets for 5 minutes. This due to some droplets was break because of the prolonged exposed to the high shear. Thus, 5 minutes emulsification time is sufficient for producing most stable emulsion.

Table 3 Effect of emulsifying time on emulsion stability.

\begin{tabular}{|c|c|c|c|c|c|}
\hline \multirow{2}{*}{$\begin{array}{c}\text { Time } \\
(\mathbf{m i n})\end{array}$} & \multicolumn{2}{|c|}{ Aqueous phase separated (\%) } & \multirow{2}{*}{$\begin{array}{c}\text { Droplet } \\
\text { count }\end{array}$} & $\begin{array}{c}\text { Droplet } \\
\text { size }(\boldsymbol{\mu m})\end{array}$ \\
\cline { 2 - 4 } & $\mathbf{1 0}$ min & $\mathbf{3 0}$ min & $\mathbf{6 0 m i n}$ & & 11.2 \\
\hline 3 & 2 & 15 & 21 & 120 & 4.9 \\
\hline 5 & 0 & 1 & 3 & 150 & 8.3 \\
\hline 10 & 2 & 15 & 20 & 100 & 6.0 \\
\hline 15 & 4 & 13 & 21 & 80 & \\
\hline
\end{tabular}

Diluent: palm oil; [Amberlite LA2]: 0.05M; $\left[\mathrm{Na}_{2} \mathrm{CO}_{3}\right.$ ]:0.5M; O/I: 3/1; homogenizer speed: 7000; Span 80: 3\% w/v; HLB: 4.3

\section{Effect of surfactant concentration}

The effect of varying surfactant concentration on the performance of water-in-oil emulsion stability was summarized in Table 4. Waterin-oil emulsion consists of $1 \%(\mathrm{w} / \mathrm{v})$ shows aqueous phase begin to separate in the first 10 minutes, indicating that at low surfactant concentration, the emulsion is unstable due to insufficient surfactant to reduce oil-water interfacial tension. This condition does not facilitate emulsion formation, which is only 70 droplets was counted and cause immediate droplet re-coalescence. Raising the surfactant concentration to $3 \%$ significantly enhanced the emulsion stability significantly, where only $1 \%$ of aqueous phase was separated after 30 minutes emulsion storage. More surfactant adsorbs at the interface between the oil membrane phase and internal phase at higher surfactant concentration, thus enhances the strength of adsorption layer and increase stability. Table 4 shows more droplets formed (150 droplets), representing sufficient surfactant was added into the system. Increasing surfactant concentration to 5 and $7 \%$ resulted in unstable emulsion. At high surfactant concentration, high amount of droplets can be formed. However, destabilization of emulsion occur from rapid coalescence between droplets. In addition, higher surfactant concentration also increase the viscosity of the emulsion which is not preferable in ELM since it can hinder mass transfer rate due to increasing interfacial resistance and interfere carrier-solute reaction at the interface [23]. A study by Joshi et al. [24] also observed destabilization of emulsion at higher surfactant. Therefore, $3 \%(\mathrm{w} / \mathrm{v})$ surfactant concentration was sufficient to form stable emulsion.

Table 4 Effect of surfactant concentration on emulsion stability.

\begin{tabular}{|c|c|c|c|c|c|}
\hline \multirow{2}{*}{$\begin{array}{c}\text { Conc. } \\
(\%)\end{array}$} & \multicolumn{2}{|c|}{ Aqueous phase separated (\%) } & \multirow{2}{*}{$\begin{array}{c}\text { Droplet } \\
\text { count }\end{array}$} & $\begin{array}{c}\text { Droplet } \\
\text { size }(\boldsymbol{\mu m})\end{array}$ \\
\cline { 2 - 4 } & $\mathbf{1 0}$ min & $\mathbf{3 0}$ min & $\mathbf{6 0 m i n}$ & & 3.6 \\
\hline 1 & 1 & 6 & 10 & 70 & 4.9 \\
\hline 3 & 0 & 1 & 3 & 150 & 3.6 \\
\hline 5 & 0 & 10 & 20 & 80 & 3.7 \\
\hline 7 & 5 & 10 & 24 & 60 & \\
\hline
\end{tabular}

Diluent: palm oil; [Amberlite LA2]: 0.05M; [Na2CO3]:0.5M; O/I: 3/1; homogenizer speed: 7000; emulsifying time: 5 min; HLB: 4.3

\section{CONCLUSION}

The results of this study showed that all parameters have a significant effect on the stability of water in palm oil emulsion. The most stable emulsion was observed at $7000 \mathrm{rpm}$ homogenizer speed, 5 minute emulsification time, and 3\% (w/v) span 80 . The emulsion observed was stable to half hour, and sufficient to perform extraction process. Thus, green solvent has potential to replace conventional toxic diluent.

\section{ACKNOWLEDGEMENT}

The authors would like to acknowledge the Ministry of Higher Education (MOHE), Universiti Teknologi Malaysia (RU Research Grant; GUP: Q.J130000.2546.12H50), and Centre of Lipid Engineering and Applied Research (CLEAR) for financial support to make this research possible.

\section{REFERENCES}

[1] N. Othman, H. Mat, M. Goto, Solvent. Extr. Res. Dev. 12 (2005) 27.

[2] Y. Hong, W. Hong, D. Han, . Biotechnol. Bioproc. E. 6 (2001) 386.

[3] S. Gupta, M. Chakraborty, Z.V.P. Murthy, J. Dispersion Sci. Technol. 34 (2013) 1733.

[4] H.R. Mortaheb, M.H. Amini, F. Sadeghian, B. Mokhtarani, H. Daneshyar, J. Hazard. Mater. 160 (2008) 582.

[5] A. Balasubramanian, S. Venkatesan, CLEAN - Soil, Air, Water. 42 (2014) 64.

[6] N.F.M. Noah, N. Othman, N. Jusoh, J. Taiwan Inst. Chem. Eng.

[7] T. Kakoi, N. Horinouchi, M. Goto, F. Nakashio, J. Membr. Sci. 118 (1996) 63.

[8] R.N.R. Sulaiman, N. Othman, N.A.S. Amin, J. Ind. Eng. Chem. 20 (2014) 3243.

[9] N. Othman, K.H. Chan, M. Goto, H. Mat, Solvent. Extr. Res. Dev. 13 (2006) 191.

[10] N. Othman, H. Mat, M. Goto, J. Membr. Sci. 282 (2006) 171.

[11] O. Zing-Yi, N. Othman, M. Mohamad, R. Rashid, Int. J. Global Warming. 6 (2014) 270.

[12] L. Zeng, Y. Zhang, Q. Liu, L. Yang, J. Xiao, X. Liu, Y. Yang, Chem. Eng. J. 289 (2016) 452.

[13] S.C. Lee, K.-S. Hyun, J. Membr. Sci. 350 (2010) 333.

[14] S.C. Lee, J. Membr. Sci. 381 (2011) 237.

[15] W.S.W. Ho and K.K. Sirkar, Membrane Handbook, Chapman \& Hall, New York: Van Nostrand Reinhold, 1992.

[16] M. Chakraborty, C. Bhattacharya, and S. Datta, Chapter 4 - Emulsion Liquid Membranes: Definitions and Classification, Theories, Module Design, Applications, New Directions and Perspectives, in Liquid Membranes, V.S. Kislik, Editor. 2010, Elsevier: Amsterdam. 141-199.

[17] N. Othman, S.N. Zailani, N. Mili, J. Hazard. Mater. 198 (2011) 103.

[18] M.C. Chow,C.C. Ho, J. Am. Oil Chem. Soc. 73 (1996) 47.

[19] S. Bjorkegren,R.F. Karimi, A Study of the Heavy Metal Extraction Process using Emulsion Liquid Membranes, Chalmers University of Technology, Sweden, 2012.

[20] V. Badgujar, N.K. Rastogi, Desalin. Water Treat. 36 (2011) 187.

[21] M.P. Jilska,W.S. Geoff, Use of Emulsion Liquid Membrane Systems in Chemical and Biotechnological Separations, CRC Press, 2008.

[22] W.S. Ho, T.A. Hatton, E.N. Lightfoot, N.N. Li, AIChE J. 28 (1982) 662.

[23] N. Othman, N. Mili, A. Idris, S.N. Zailani, Sustain. Membr. Technol. Energy Water Environ. (2012) 221.

[24] H.C. Joshi, I.P. Pandey, A. Kumar, N. Garg, Adv. Pure Appl. Chem. 1 (2012) 7 . 